\title{
Médiévales
}

Langues, Textes, Histoire

66 | printemps 2014

Harmonie Disharmonie

\section{Figures de l'harmonia mundi dans les manuscrits et les stalles gothiques en France}

Le roi David accordant sa harpe et les anges musiciens

Representations of the harmonia mundi in Gothic Manuscripts and Choir Stalls in France. King David Tuning his Harp and Angel Musicians

Welleda Muller

\section{OpenEdition}

Journals

Édition électronique

URL : https://journals.openedition.org/medievales/7190

DOI : 10.4000/medievales. 7190

ISSN : 1777-5892

Éditeur

Presses universitaires de Vincennes

Édition imprimée

Date de publication : 30 juin 2014

Pagination : 43-63

ISBN : 978-2-84292-405-8

ISSN : 0751-2708

Référence électronique

Welleda Muller, "Figures de l'harmonia mundi dans les manuscrits et les stalles gothiques en France», Médiévales [En ligne], 66 | printemps 2014, mis en ligne le 05 juillet 2016, consulté le 24 avril 2022.

URL : http://journals.openedition.org/medievales/7190; DOI : https://doi.org/10.4000/medievales. 7190

Tous droits réservés 
Welleda Muller

\title{
Figures de l'harmonia mundi dans les manuscrits et les stalles gothiques en France
}

\author{
Le roi David accordant sa harpe et les anges musiciens
}

La musique est très présente dans les images médiévales, que ce soit à travers les représentations de musiciens ou, de façon plus subtile, par l'emploi des mêmes proportions mathématiques que celles des intervalles musicaux ${ }^{1}$. Les anges musiciens sont nombreux à être figurés sur tout type de support artistique. À côté de ces figures spirituelles, le musicien le plus récurrent est le roi David. Or, si ces deux exemples de musiciens sont assez différenciés et expriment une musique qui leur est propre, ils participent tous deux à une même vision de l'harmonie du monde créé par Dieu. Transmise aux théoriciens médiévaux par les courants pythagoricien et platonicien, la notion d'harmonie regroupe des phénomènes acoustiques et dynamiques mais aussi des réflexions intellectuelles sur le monde. Dieu a créé le monde suivant des proportions mathématiques parfaitement harmonieuses et ce monde résonne au-delà des sens humains ${ }^{2}$; néanmoins les êtres supérieurs jouant de la musique comme le roi David ou les anges sont si nombreux dans les images que l'on peut se demander s'ils ne sont pas la matérialisation visuelle de la musica mundana. Depuis Boèce (470-525), celle-ci fait partie, avec la musica humana (harmonie du corps, de l'âme et de l'esprit humain) et la musica instrumentalis (art des sons vocaux et instrumentaux), d'un tout exprimant l'harmonie de la divine Création (De institutione musica, I, 2). Si la musique des sphères ne résonne pas aux oreilles des hommes, comme l'expliquent de nombreux théologiens ${ }^{3}$, le besoin de représenter ce son semble toutefois émerger au Moyen Âge, avec les «moyens» à la

1. Cf.C.RAult, Géométrie médiévale, tracés d'instruments et proportions harmoniques, Royaumont, 1994; I. Marchesin, L'Image organum. La représentation de la musique dans les psautiers médiévaux. 800-1200, Turnhout, 2000.

2. Cf. MAcrobe, In Somnium Scipionis, II, 4, 14-15 et BoÈcE, De Institutione Musica, I, 2.

3. Sur le topos de l'harmonie des sphères chez les théologiens voir I. MARCHESIN, L'Image organum..., p. 41-42. 
disposition des artistes: les instruments de musique dans les mains d'êtres spirituels comme les anges ou de personnages emblématiques à la valeur exemplaire tel le roi David.

Afin d'élaborer une hypothèse de compréhension de l'expression de l'harmonie du monde dans l'art médiéval, nous examinerons les images d'anges musiciens et du roi David accordant sa harpe, car si le monde créé par Dieu était d'une harmonie parfaite à l'origine, il est nécessaire d'ajuster cette harmonie voire de la retrouver lorsqu'on l'a perdue ${ }^{4}$. Le corpus réuni pour cette étude est circonscrit à la sculpture des stalles de chœur et à la peinture des manuscrits liturgiques dans la France gothique, de la fin du XII ${ }^{\mathrm{e}}$ siècle jusqu'à l'orée du $\mathrm{XVI}^{\mathrm{e}}$ siècle ${ }^{5}$; deux supports qui ont pour caractéristique la juxtaposition du son et de l'image: les moines et les chanoines chantaient dans les stalles (dont la structure était étudiée pour une acoustique optimale) et les manuscrits étaient les supports privilégiés du chant, même lorsqu'ils ne comportaient pas de notation musicale ${ }^{6}$. Nous verrons qu'avec l'avènement de la Renaissance, un changement de paradigme se produit dans les conceptions du monde; évolution visible dans les représentations de l'harmonie.

\section{Le roi David accordant sa harpe}

Dans le paysage iconographique médiéval, la figure du roi David domine $^{7}$ et fait office de modèle pour tous les musiciens. Les inscriptions juxtaposées aux images des psautiers romans le qualifient presque toujours de poète ou de psalmiste et de musicien ou d'artiste ${ }^{8}$. Sa double capacité d'instrumentiste et d'auteur littéraire est ainsi soulignée et portée aux nues, en particulier dans les enluminures de manuscrits. Mais plus qu'une fonction de modèle ${ }^{9}$ pour les musiciens médiévaux, le roi David exprime

4. Saint Augustin écrit que cette harmonie n'est pas complètement perdue, puisqu'elle a laissé des indices de son existence dans la liturgie chantée; $c f$. son De Musica, VI, XI, 30.

5. Notons que les premières stalles historiées conservées aujourd'hui en France datent de la fin du XIV $\mathrm{s}$.

6. Sur les questions de notations musicales dans les manuscrits, $c f$. notamment S. Corbin, «La notation musicale», dans Histoire de la musique, Paris, 1960, t. I, p. 691692; M. Huglo, Les Livres de chant liturgique, Turnhout, 1988; N. Bell, Music in Medieval Manuscripts, Londres/Toronto, 2001; M.-N. COLETTE et alii, Histoire de la notation musicale Manuscripts, Londres/Toronto, 2001; M.-N. Co
du Moyen Âge à la Renaissance, Paris, 2003.

7. Le roi David est le personnage de la Bible le plus souvent représenté au Moyen Âge et ses représentations en musicien sont innombrables: $c f$. notamment H. Steger, David Rex et Propheta. König David als vorbildliche Verkörperung des Herrschers und Dichters im Mittelalter, nach Bilddarstellungen des achten bis zwölften Jarhunderts, Nuremberg, 1961.

8. I. MARCHESIN, L'Image organum..., p. 19-20.

9. Reprenant une expression forgée par J. Baschet, M. Clouzot parle d'«imagehyperthème» pour qualifier les représentations du roi David musicien: Le Jongleur. Mémoire aussi l'harmonie créée par Dieu, et il est représenté en figure ordonnatrice du monde à travers les images le figurant en musicien. Il n'existe que peu de variations iconographiques de cette figure entre le XIII et le $\mathrm{XV}^{\mathrm{e}}$ siècle. On trouve une douzaine de représentations de David musicien dans les stalles française ${ }^{10}$; certaines sont sculptées sur les dorsaux, et présentent le roi au sein d'un cortège de saints. C'est le cas à Cuiseaux (Saône-et-Loire, Bourgogne) où il ouvre le cortège des apôtres ; à Saint-Claude (Jura,FrancheComté) (fig. 1), il est en alternance avec des prophètes et des apôtres, au sein d'un double Credo ${ }^{11}$. Dans ces représentations, il est très rare que le roi joue de son instrument; la harpe, qui est son attribut instrumental le plus courant à l'époque gothique, est posée au sol près de lui, parfois même encore dans sa housse (Estavayer, Suisse). L'harmonie exprimée alors ne résonne pas; c'est par la posture et l'attitude de David, mais aussi par la présence d'un instrument à la fois attribut du roi et évocateur de l'harmonie du monde, que l'univers harmonieux créé par Dieu est évoqué par métonymie. Parce qu'il ne joue pas de son instrument, David fait référence à l'harmonie du monde qui ne résonne pas aux oreilles humaines ${ }^{12}$ et ne passe pas par des instruments de musique «artificielle».

Outre ses apparitions dans les cortèges de saints, le roi David est également sculpté dans l'arbre de Jessé sur les stalles. Dans ce cas, l'instrument de David est assez systématiquement joué: le roi est assis dans les rinceaux végétaux de l'arbre et il pince les cordes de sa harpe (Saint-Cernin, Cantal, Auvergne). Un exemple très intéressant, en dehors du corpus de cette étude, est celui d'Erfurt (Allemagne) dans lequel le roi accorde sa harpe avec une clef d'accord, représentation très courante dans les enluminures mais beaucoup plus rare dans les stalles. Or, cette spécificité iconographique est d'autant plus importante que David est représenté au sommet de l'arbre de Jessé dans lequel jouent également des anges musiciens. David est alors représenté en «accordeur» de la musique spirituelle des anges; il restaure l'harmonie créée par Dieu.

Si David joue rarement de sa harpe dans la sculpture des stalles, en revanche cet instrument a un rôle d'attribut, qui suffit à lui seul pou

de l'image au Moyen Âge. Figures, figurations et musicalité dans les manuscrits enluminés (1200-1330), Berne, 2011, p. 140-141.

10. Répertoriées dans la base de données Musicastallis : http://www.plm.paris-sorbonne. $\mathrm{fr} /$ musicastallis.

11. L'iconographie du Credo est particulièrement répandue dans le duché de Savoie: $c f$. C. Charles, Stalles sculptées du XVe siècle. Genève et le duché de Savoie, Paris, 1999 p. 21-30.

12. Macrobe écrit par exemple: «Si la musique engendrée par la perpétuelle révolution du ciel ne nous est pas audible en une claire perception, c'est que le son en excède l'étroi canal des oreilles humaines [...]. La perception des autres hommes ne peut saisir par l'ouïe l'harmonie universelle» (Commentaire au Songe de Scipion, II, 4, 14-15, éd. et trad. M. Armisen-Marchetti, Paris, t. II, 2003, p. 21). 


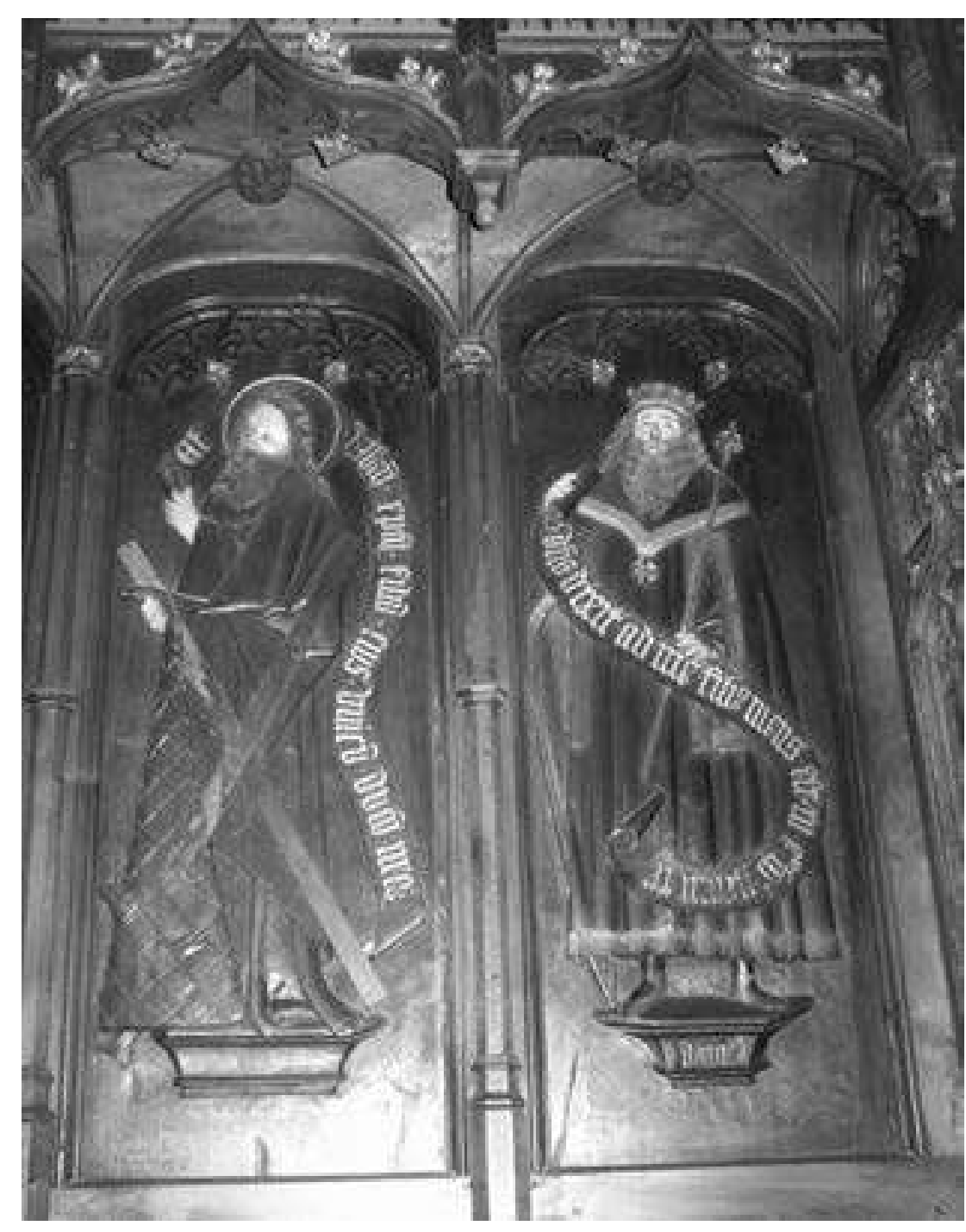

Fig. 1. Dorsaux des stalles de Saint-Claude (ㅇ W. Muller).

identifier le personnage. Ainsi, à Soignoles-en-Brie (Seine-et-Marne, Îlede-France), le jeune David est figuré face à deux lions: la petite harpe posée à ses pieds permet aussi clairement qu'une inscription de reconnaitre David et, par voie de conséquence, la scène représentée. Idem à Aarschot (Belgique), lorsqu'il s'agenouille devant l'apparition de l'Arche d'Alliance. Notons que ces exemples sont relativement tardifs (1500-1530); fallait-il expliciter à cette époque charnière une iconographie qui auparavant était évidente? Le début du $\mathrm{XvI}^{\mathrm{e}}$ siècle est en effet marqué par une mouvance illustrative dans les stalles, alors que leur iconographie au siècle précédent était beaucoup plus $«$ symbolique ${ }^{13}$ ».

Globalement, les images sculptées du roi David jouant de la harpe dans les stalles françaises datent souvent du XVI ${ }^{e}$ siècle. Bien que la majorité des ensembles de stalles en Europe date du Xve siècle, du fait de la destruction d'ensembles plus anciens, il semblerait que les personnages bibliques ne fassent leur apparition que tardivement sur ce support, à l'iconographie plus volontiers «profane» aux XIV et XV siècles. Il faut d'ailleurs sortir de France pour trouver des images du roi David musicien du XIV siècle; les plus anciens exemples sont à Erfurt (Allemagne, 1370), Chester (GrandeBretagne, 1380) et Bamberg (Allemagne, 1380-1400).

La fourchette chronologique est plus large pour les images du roi David musicien dans les enluminures de manuscrits français. La base de données Initiale de l'IRHT ${ }^{14}$ recense une centaine d'exemples de David jouant d'un instrument à cordes ${ }^{15}$ dans les manuscrits produits en France datant de la fin du $\mathrm{Xl}^{\mathrm{e}}$ au début du XVI $\mathrm{I}^{\mathrm{e}}$ siècle, majoritairement des ouvrages liturgiques, dans lesquels le roi illustre généralement des folii relatifs aux psaumes. On peut ajouter à cela une quarantaine d'images de David sonnan des cloches, iconographie qui ne semble en revanche pas remonter avant le $\mathrm{XIII}^{\mathrm{e}}$ siècle dans le corpus. D'une manière générale on peut remarquer que les instruments tenus et joués par le roi David varient peu dans les manuscrits, puisqu'à partir du XIII siècle l'écrasante majorité est en effet constituée de la harpe et des cloches; et lorsque le roi est entouré de plusieurs instruments, c'est presque systématiquement la harpe (Bible historiale, ms. Paris, $\mathrm{BnF}$, fr. 9, fo 283) ou les cloches (Bréviaire à l'usage de Paris, ms. Châteauroux, $\left.\mathrm{BM}, 2, \mathrm{f}^{\circ} 48 \mathrm{v}^{\circ}\right)$ qu'il choisit de faire résonner. De nombreux manuscrits comportent d'ailleurs deux images du roi David musicien, l'une le montrant jouant ou accordant sa harpe majoritairement dans l'initiale B du premier

13. Sur l'évolution de l'iconographie des stalles entre la fin du xiv' s. et le concile de Trente, en particulier dans le Grand Duché de Bourgogne, $c f$. W. MulLer, Les Stalles, siège du corps dans les chœurs liturgiques du Grand Duché de Bourgogne aux XV' et XVI siècles, Turnhout, 2014, partie 4, chap. 2.

14. http://initiale.irht.cnrs.fr/accueil/index.php.

15. La harpe est le cordophone plus fréquent, mais on trouve aussi la vièle et le psaltérion. 
psaume ${ }^{16}$, l'autre le présentant sonnant un jeu de cloches suspendues (ou tintinnabulum ${ }^{17}$ généralement dans l'initiale $\mathrm{E}$ du psaume $81^{18}$.

Ces images illustrent des manuscrits liturgiques; des psautiers comme on pouvait s'y attendre, mais également des bréviaires et des Bibles, où le roi David harpiste est souvent juxtaposé avec le jeune David décapitant Goliath (Bible, ms. Alençon, BM, 54, fo 170). Lorsque David est dans une lettrine, il s'agit le plus souvent de l'initiale B du premier psaume (ou de l'initiale E du psaume 81 lorsqu'il joue des cloches). On imagine que, outre la surface offerte par le B et l'importance du premier psaume, le texte lui-même: «Heureux l'homme qui ne marche pas selon le conseil des méchants, qui ne s'arrête pas sur la voie des pécheurs, et qui ne s'assied pas en compagnie des moqueurs » a certainement inspiré cette image du roi David musicien qui est ainsi inscrit dans l'ordre harmonieux du monde et offre un exemple à ceux qui verront l'enluminure. Et ce modèle semble renforcé lorsqu'il est juxtaposé avec David combattant Goliath (Bible, ms. Besançon, BM, 4, fo 238 ), puisque la violence du combat est systématiquement représentée en dessous, hiérarchisée par rapport à la figure de David musicien. Or, dans la plupart de ces exemples, David ne fait pas que jouer de la harpe, il l'accorde grâce à une clef d'accord (Bible, ms. Paris, BnF, lat. 17198, fo $\left.{ }^{\circ} 195\right)^{19}$.

Sur la petite centaine de représentations de David jouant d'instruments à cordes recensée par Initiale, on dénombre une quarantaine d'images montrant l'accord de la harpe, soit presque la moitié des exemples: d'où l'importance de cette iconographie spécifique, pourtant peu relevée par les spécialistes jusqu'à présent ${ }^{20}$. La clef d'accord est parfois mise en valeur, certainement pour que l'action du roi soit évidente et magnifiée (Bible, ms. Avranches, BM, 3, $2^{\mathrm{e}}$ vol., fo 3 ). Beaucoup d'images montrent le roi penchant la tête vers sa harpe, mettant ainsi en évidence cette action de l'accord de l'instrument et l'importance de l'écoute de l'harmonie (Comment. in

16. «Beatus vir qui non abiit in consilio $[\ldots] »$ (Psaume 1.1)

7. Si la harpe est mentionnée dans plusieurs psaumes $(6.1,12.1,33.2,43.4,49.4,57.8$, $71.22,81.2,92.3,98.5,108.2,147.7,149.3,150.3)$, ce n'est pas le cas des cloches; or, la présence de celles-ci sonnées par David pourraient être l'évocation d'un lien privilégié entre le roi et Pythagore qui, selon Boèce, découvrit les proportions musicales régissant l'harmonie du monde en passant près d'une forge où différents marteaux frappaient des enclumes produisant ainsi des sons différents comme avec des cloches: $c f$. BoÈcE, De institutione musica I I 10. Cette collusion renverrait alors encore une fois à l'idée d'harmonie du monde à travers l'évocation de la légende de Pythagore et des cloches.

18. «Exultate Deo adjutori nostro [...]» (Psaume 81.2-4)

19. Notons que cette action semble dévolue à la harpe, puisqu'il ne semble pas exister de cas où David accorderait un autre instrument à cordes, tel le psaltérion dont il joue parfois.

20. Mis à part le roi David, je n'ai rencontré qu'un seul autre personnage accordant un instrument dans l'art gothique, Iseult: $c f$. W. MuLLeR, «Tristan et Iseult illustrés dans les stalles anglaises. L'irruption d'images profanes dans un contexte sacré», dans les actes du colloque Tristan et Yseut, l'éternel retour, éd. D. BusChINGER, Bulletin du CEM d'Amiens, à paraitre.
Psalmos, ms. Troyes, BM, 58, fo 3 ; Comment. in Psalmos, ms. Angers, BM 051, fo 3 ; Bréviaire prémontré, ms. Charleville-Mézières, BM, 50, fo 1). Quelques exemples viennent apporter un caractère symbolique encore plus fort à ces images, avec l'intervention du Saint-Esprit sous la forme d'un oiseau, comme on peut le voir dans le $\mathrm{f}^{\circ} 130 \mathrm{v}^{\circ}$ de la Bible d'Angers (Bible, ms. Angers, BM, 9) (fig. 2) le Saint-Esprit se pose sur la clef et semble chuchoter à l'oreille de David la bonne harmonie à régler. On trouve une scène similaire dans la Bible d'Avranches (Bible, ms. Avranches, BM, 3 , $\mathrm{f}^{\circ} 3$ ). Ces images nous apprennent que l'harmonie est un état du monde, de la Création, qui a besoin d'être retrouvée, et le réglage des instruments de musique en est une bonne métaphore. Le roi David, parfois guidé par le Saint-Esprit, cherche cette harmonie et représente ainsi un modèle pour les fidèles, qui participent à la liturgie en étant assis dans les stalles (ou dans la nef en ce qui concerne les laïcs) et dont les manuscrits liturgiques sont un des supports pour retrouver l'ordre harmonieux du monde créé par Dieu ${ }^{21}$ Isabelle Marchesin ajoute que cette image fait écho à la figure du Christ et, par là, à l'homme nouveau, qui a la possibilité de se «placer en accord, en consonance avec la loi divine, grâce à la présence fondamentale en lui de l'Esprit saint $[\ldots]^{22} »$. Outre cette évocation, la musique du roi David exprime aussi l'harmonie du monde par son fort potentiel d'apaisement des âmes, puisque lorsqu'il joue de la harpe pour le roi Saül, celui-ci «se trouvait soulagé, et le mauvais esprit se retirait de lui ${ }^{23}$ ».

Cette idée de David en tant que modèle pour les fidèles est renforcée par l'existence d'un petit nombre d'images dans lesquelles le roi est figuré aux côtés de chantres. Ainsi, au fo 133 du manuscrit Paris, BnF, lat. 774 (datant de la fin du $\mathrm{Xv}^{\mathrm{e}}$ siècle), le roi est représenté tenant sa harpe encore à demi-enveloppée dans sa housse, derrière un groupe de quatre chantres chantant la musique notée sur un livre posé sur un lutrin. Le texte en dessous de la miniature est celui du psaume $96^{24}$ qui incite à chanter des cantiques au

21. Voir les travaux d'É. Palazzo sur les cinq sens et leur activation par la liturgie et le chant (noté dans les manuscrits) dans le but de retrouver l'harmonie: "Les cinq sen au Moyen Âge. État de la question et perspectives de recherche», Cahiers de Civilisation Médiévale, 55 (2012), p. 339-366; Art Liturgy and the Five Senses in the Early Middle A dies, Viator, 41 Ages», Vialor, 41 (2010); «La dimension sonore de la liturgie dans l'Antiquité et au Moye Âge », dans Archéologie du son. Les dispositifs de pots acoustiques dans les édifices anciens, Paris, 2012, p. 51-58.

22. Moyen Age, entre ordre et désordre, Paris, 2004, p. 82. 21 $-2)$. 


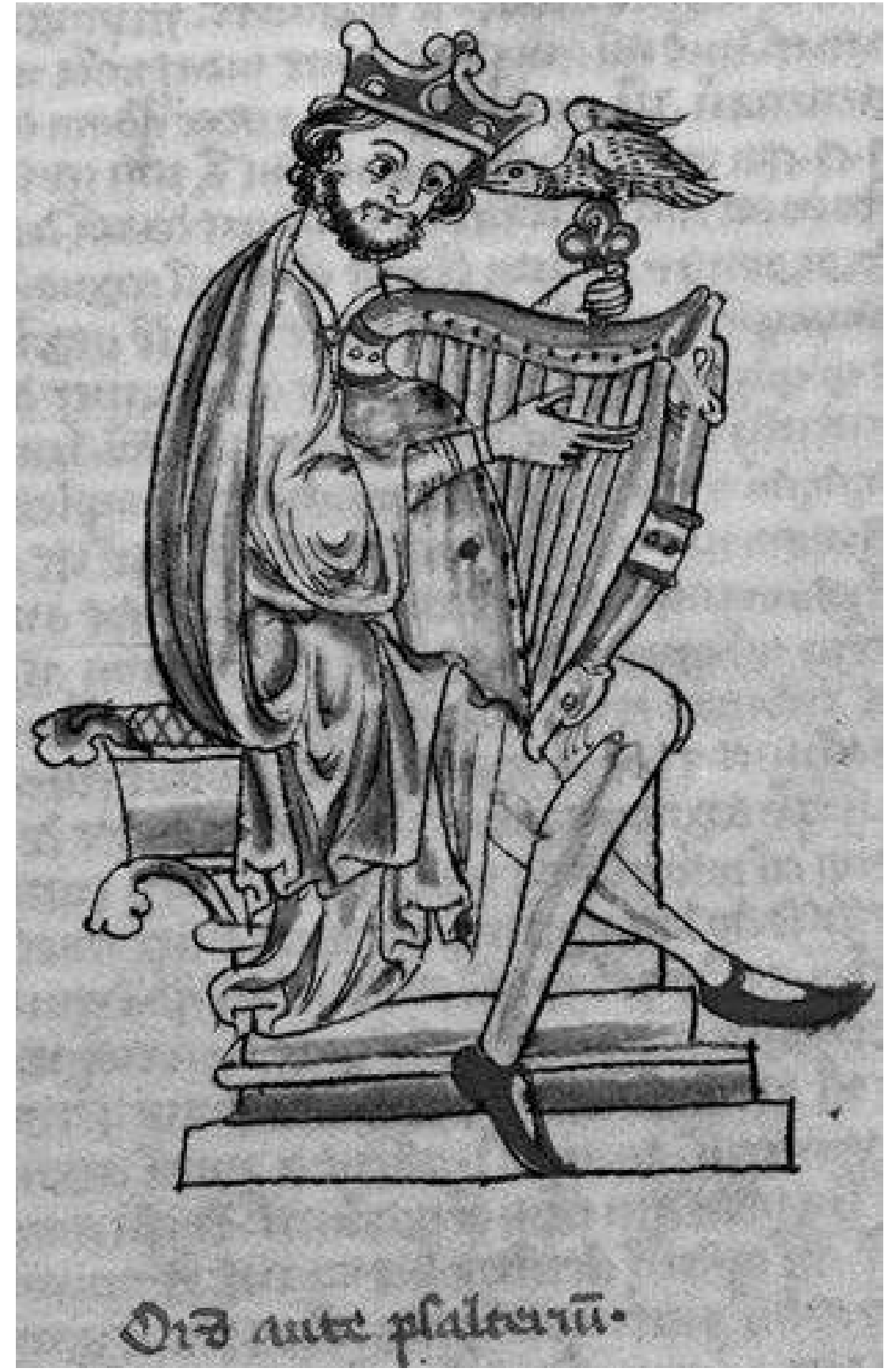

Fig. 2. David accordant sa harpe, BM d'Angers, ms. 9, $\mathrm{f}^{\circ} 130 \mathrm{v}^{\circ}$ (C) BM d'Angers, IRHT).
Seigneur pour trouver le Salut ${ }^{25}$. Le roi David accordant sa harpe est alors l'image d'une figure ordonnatrice du monde, miroir du Christ et de l'homo novus, dépouillé du vieil homme et lavé du péché originel, exemple pour les fidèles qui suivent le modèle qu'il offre en cherchant à retrouver l'harmonie perdue par la liturgie qui s'exprime presque exclusivement en musique à l'époque qui nous occupe ${ }^{26}$. Chaque chantre est certainement invité par ces images à accorder son âme à Dieu et à se mettre en consonance par rapport à la Création divine, à réorganiser son cœur (chœur) dans l'harmonie du monde grâce à la pratique de la liturgie.

\section{Les anges musiciens}

À côté des images du roi David, les anges sont aussi très souvent représentés jouant d'instruments de musique assez divers. Si l'iconographie médiévale comporte une multitude d'anges, les anges musiciens sont particulièrement nombreux et ce sur tous les supports artistiques: stalles et manuscrits, sculpture monumentale, vitraux, ivoires sculptés, tapisseries, etc Les figures angéliques sont présentes dans l'Ancien comme dans le Nouveau Testament, mais la culture médiévale est aussi marquée par la Hiérarchie céleste du Pseudo-Denys l'Aréopagite ${ }^{27}$. Écrit au vi siècle et abondamment copié jusqu'à la fin du Moyen Âge, cet ouvrage classe les anges en trois hiérarchies de trois chœurs. Et le mot chœur n'est pas anodin ${ }^{28}$, puisque les anges participent activement à l'harmonie du monde créé par Dieu. On sait que dans les conceptions médiévales du monde, les anges permettent aux orbes de se mouvoir ${ }^{29}$ et c'est de ce mouvement que naît l'harmonia mundi; ils ont alors une fonction essentielle au sein du cosmos, ce qui explique peut-être le nombre si important d'anges musiciens dans l'art médiéval en général et dans les stalles et les manuscrits en particulier.

Les anges musiciens sont présents dans les stalles françaises de la fin du XIV $v^{\mathrm{e}}$ jusqu'au milieu du $\mathrm{XVI}^{\mathrm{e}}$ siècle, en nombre à peu près

25. On trouve aussi David jouant du psaltérion pour accompagner des chantres avec l'aide de deux musiciens au folio $154 \mathrm{v}^{\circ} \mathrm{du}$ ms. Paris, BnF, lat. 10491 (Diurnal de René II de Lorraine, 1492-1493).

26. Cf. É. Palazzo, Liturgie et société au Moyen Âge, Paris, 2000, chap. 1.

27. Pseudo-Denys l'Aréopagite, La Hiérarchie céleste, trad. M. de Gandillac, Paris, 1958 .

28. Terme employé dans les traductions latines de l'ouvrage du Pseudo-Denys en circulation au Moyen Âge (mais pas dans l'original en grec).

29. Parmi tant d'autres, citons Dante Alighieri, Convivio II, IV, 2, et Thomas d'Aquin, Somme théologique, I, 115, 4 ; cf. aussi T. SuAREZ-NANI, Les Anges et la philosophie, Paris, 2002, p. 137 sq. 
équivalent ${ }^{30}$. Toutefois, on remarque qu'ils se raréfient au $\mathrm{XVI}^{\mathrm{e}}$ siècle, et après le Concile de Trente ils ont disparu. Dans les différents exemples recensés dans le corpus des stalles françaises, il est remarquable que les instruments privilégiés par les anges sont majoritairement des cordophones, qui font partie de la famille des bas-instruments ${ }^{31}$. Sur environ quatre-vingts représentations d'anges dans les stalles françaises, une quarantaine utilise des cordophones, une trentaine des aérophones ${ }^{32}$ et on compte seulement quatre anges musiciens tenant des idiophones (surtout des triangles) et cinq tenant des membranophones (généralement des tambourins) (fig. 3). Précisons toutefois qu'il est difficile d'établir des statistiques pertinentes étant donné les nombreuses destructions de stalles en France.

Le luth est certainement l'instrument le plus répandu entre les mains des anges ${ }^{33}$. Il est représenté avec réalisme par les huchiers ${ }^{34}$ et ces anges luthistes semblent exprimer tout particulièrement l'ordre: ils sont assis, ne gesticulent pas comme les jongleurs ou les animaux, pincent les cordes parfois groupées en chœurs avec les doigts (Venable, Eure, HauteNormandie) ou avec un plectre ${ }^{35}$. Viennent ensuite les différentes sortes de vièles à archets, la plupart du temps tenues sur l'épaule et non entre les jambes $^{36}$.

Si les anges sont aussi les annonciateurs du Jugement Dernier en soufflant dans des trompettes, ce genre d'instrument bruyant est rarement présent entre leurs mains dans les stalles; en revanche on en trouve quelques exemples dans les enluminures de manuscrits en dehors des images du Jugement Dernier où ils sont évidemment les plus nombreux (Psautierheures, ms. Beaune, BM, 39, fo $\left.174 \mathrm{v}^{\circ}\right)$. Ainsi dans le Psautier-heures conservé à la bibliothèque municipale d'Avignon (ms. 121, fo $\left.151 \mathrm{v}^{\circ}\right)$, on trouve au début du psaume $80^{37}$, dans un petit édicule, deux anges sonnant des cloches surmontés de deux anges trompettistes. Ces quatre anges semblent illustrer les premiers versets du psaume 80 qui exhortent Dieu à

30. Le nombre d'ensemble de stalles datant d'avant le xIV ${ }^{\mathrm{e}} \mathrm{s}$. étant très rare, il est difficile de trancher sur l'époque de l'apparition des anges musiciens dans les stalles en France.

31. Qualification poétique qui, par opposition aux hauts instruments, désigne les instruments à faible volume sonore qui permettaient d'accompagner la voix, notamment dans la liturgie: $c f$. L Charles-Dominieue, Musiques savantes, musiques populaires. Les ans la liturgie: $c f$. L. ChARLES-DominiQue, Musiques savantes, musiques populaires. Les

32. Les orgues portatifs étant inclus dans cette catégorie.

33. Une vingtaine dans le corpus.

34. Facteurs de stalles, chargés à la fois de la menuiserie et de la sculpture.

35. Il ne semble pas y avoir d'exemple en France, on en trouve un à Chester (GrandeBretagne).

36. Les exemples d'instruments tenus entre les jambes sont très rares dans l'art médiéval en général, et absents des stalles françaises en particulier.

37. «Prête l'oreille, berger d'Israël, toi qui conduis Joseph comme un troupeau ! prêter l'oreille à l'appel des hommes, d'où peut-être la mise en valeur d'un son puissant: celui des trompettes et des cloches conjugué.

Comme dans les stalles, ce sont les cordophones qui dominent l'iconographie angélique des enluminures. Illustrant majoritairement des bréviaires, ces anges musiciens apparaissent dans des lettrines (Bible, ms. Reims, BM, 42, $\mathrm{f}^{\circ} 126 \mathrm{v}^{\circ}$ ) ou en marges de scènes du Nouveau Testament (Bible, ms. Reims, BM, 41, fo 162), telle l'Annonciation (Psautier-heures, ms. Avignon, BM, 121, fo 7). Si leur nature angélique est évidente, le cas des marges de manuscrits est problématique, puisque des créatures ailées à buste humain et arrière-train animal ou monstrueux apparaissent aussi jouant d'instruments de musique, à l'instar des anges (Bréviaire à l'usage de Verdun, ms. Verdun, $\left.\mathrm{BM}, 107, \mathrm{f}^{\circ} 6 \mathrm{v}^{\circ}\right)$. Les hybrides sont évidemment une constante de l'iconographie marginale ${ }^{38}$, mais ce cas d' «anges mutants» pose la question de l'expression d'une musique qui n'exprime plus l'harmonie du monde et la musique des sphères, mais bien une musique déviante qui est plus attachée à la notion de désordre, étant donné la valeur transgressive de ces créatures ${ }^{39}$. Néanmoins, étant donné la polysémie de sens des images médiévales, on pourrait aussi voir, dans la prolifération de ces anges-hybrides musiciens, une référence à la République de Platon dans laquelle l'âme est décrite comme une sorte de chimère (IX, 588b), un monstre à formes multiples qui peut devenir humain par le rétablissement de l'harmonie dans le corps de l'être humain «en vue de maintenir l'accord dans son âme» (IX, 591c). Peut-être s'agit-il aussi d'une référence aux sirènes platoniciennes assises sur les cercles de l'univers, chacune faisant résonner une note différente, formant un accord harmonieux (Rép. $X, 617 b)$, mais il est plus difficile d'en être certain étant donné la forte valeur transgressive des hybrides dans le contexte médiéval. Ces créatures musiciennes seraient alors plutôt des images de l'harmonie perdue que l'être humain doit retrouver.

Outre les citoles, guiternes, vièles et psaltérions, l'orgue positif ou portatif est un des instruments très fréquemment représenté aux mains des anges dans les enluminures (Bréviaire à l'usage de Paris, ms. Châteauroux, $\mathrm{BM}, 2, \mathrm{f}^{\circ} 323 \mathrm{v}^{\circ}$ ) comme dans les stalles (jouée, cathédrale d'Amiens, Somme, Picardie [fig. 3]). Or, nous savons que cet instrument jouissait d'une symbolique positive au Moyen Âge ${ }^{40}$; son maniement complexe

38. Cf. M. CAMILLE, Images dans les marges. Aux limites de l'art médiéval, Paris, 1997 J. Wirth éd., Les Marges à drôleries des manuscrits gothiques, Genève, 2008, p. 228 sq.

39. Ces créatures sont en effet de purs hybrides, monstrueuses parce qu'elles transgressen la création divine: $c f$. G. Bartholeyns, P.-O. DitTMAR, V. Jolivet, Image et transgression au Moyen Âge, Paris, 2008, p. $26 s q$.; $c f$. aussi W. MulLeR, «Hybrids in Choirstalls, a Myth Transgressed or Aristotle Denied ?», Porticvm, 4 (2012), p. 80-88.

40. Cf. L. Charles-Dominioue, "Classer les instruments de musique: entreprise rationaliste ou pensée symbolique», Prétentaine, 22 (novembre 2007), p. 191-215. 


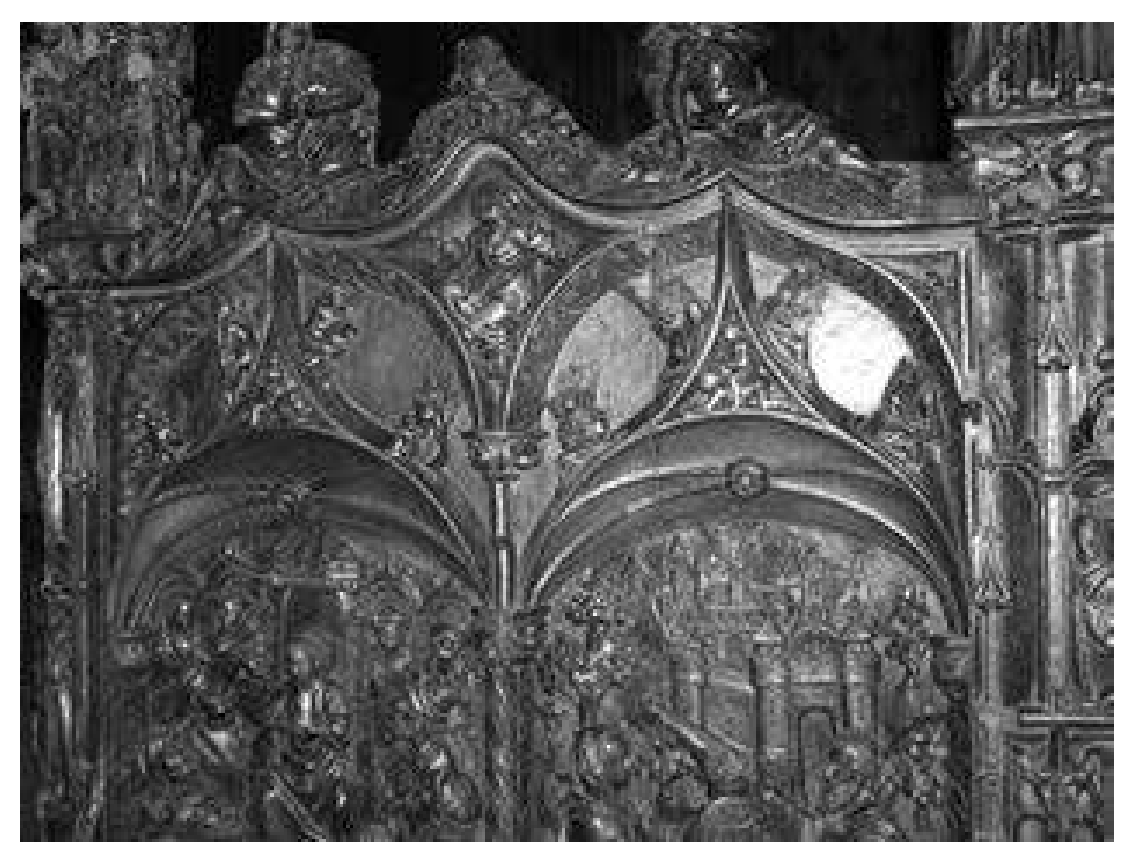

Fig. 3. Anges et putti musiciens, haut d'une jouée des stalles de la cathédrale d'Amiens (ㅇ W. Muller)

(car polyphonique) impliquait une connaissance de la théorie musicale, ce qui valorisait l'instrumentiste capable d'en jouer; en outre, de faible volume sonore, il permettait d'accompagner le chant dans la liturgie et, dès la fin $\mathrm{du} \mathrm{Xv}^{\mathrm{e}}$ siècle et surtout au siècle suivant, il deviendra fixe dans les édifices religieux. Cet instrument n'est d'ailleurs pas souvent joué par des instrumentistes humains, que ce soit dans les stalles comme dans les enluminures; toutefois, il est remarquable que l'organetto soit l'apanage des femmes (stalles de Saint-Chamant, Cantal, Auvergne; miniature du Psautier-heures, ms. Avignon, BM, 121, fo 95), notamment sur des objets en ivoire sculpté ${ }^{41}$. En dehors du contexte courtois, les femmes organistes sont souvent des allégories, comme dans l'exemple du Psautier-heures conservé à la bibliothèque municipale d'Avignon (ms. 121, fo 95 ), où une figure féminine accompagne le roi David jouant de la vièle et fait figure d'allégorie de l'Ecclesia face à la Synagogua symbolisée par David.

41. Citons deux exemples de valves de miroir du XIV $\mathrm{e}$. dans lesquelles une femme jouant de l'orgue portatif accompagne un groupe de nobles; l'une est conservée à l'Art Gallery of Ontario (Toronto), l'autre au musée du Louvre (Paris); ces objets sont visibles sur le site du Gothic Ivories Project: http://www.gothicivories.courtauld ac .uk.
Notons que, mis à part un exemple dans le Graduel à l'usage de l'abbaye de la Trinité de Vendôme (ms. Vendôme, BM, 267, fo $188 \mathrm{v}^{\circ}$ ), ces anges musiciens ne sont que rarement juxtaposés à de la notation musicale; nombreux dans les bibles et les bréviaires, ils sont en effet relativement absents des graduels et des antiphonaires. Alison Stones a remarqué que ce type de manuscrits liturgiques des $\mathrm{XII}^{\mathrm{e}}$ et $\mathrm{XIII}^{\mathrm{e}}$ siècles comportant de la notation musicale est souvent dépourvu d'iconographie musicale ${ }^{42}$. Peutêtre cette absence est-elle à mettre en relation avec une liturgie chantée dont l'accompagnement instrumental, s'il est avéré, pose problème à de nombreux théologiens ${ }^{43}$.

Certains instruments sont bannis, ou presque, des mains des anges C'est le cas de la cornemuse qui, à l'exception de rares exemples (que l'on peut plutôt qualifier de «Renaissance», comme c'est le cas dans les stalles du Mans et dans le Bréviaire à l'usage de Paris, ms. Châteauroux, $\mathrm{BM}, 2$, fo $349 \mathrm{v}^{\circ}$ ), est plutôt l'apanage des animaux (Oviedo, Espagne) des bergers (Rouen, Seine-Maritime, Haute-Normandie) ou des musiciens professionnels (Amiens, Somme, Picardie) et des fous (Poulaines, Indre, Centre $^{44}$ ). La symbolique sexuelle de la cornemuse, de par sa ressemblance avec les parties génitales masculines ${ }^{45}$, ainsi que son rattachement à la musique diabolique ${ }^{46}$, induisent la rareté de son utilisation par les anges ${ }^{47}$. Le fait que les anges utilisent beaucoup plus souvent des cordophones que des aérophones (l'orgue mis à part) dans les images médiévales renvoie aussi certainement à l'opposition entre la corde et le vent existant depuis l'Antiquité avec le Jugement de Midas qui préféra la lyre d'Apollon à la flûte de $\operatorname{Pan}^{48}$. Outre l'opposition entre instruments hauts et instruments bas évoquée précédemment, la corde est très souvent valorisée par rapport au vent. Luc Charles-Dominique relève par exemple que les aérophones ont un lien privilégié avec la folie et l'érotisme, alors que les cordophones sont associés au Christ en croix car ils sont constitués d'une caisse de bois sur

42. A. Stones, «Iconographie des Graduels et Antiphonaires des XII et XIII ${ }^{\mathrm{e}}$ s.» intervention au séminaire Musiconis, 29 mars 2012, Université Paris-Sorbonne.

43. Notamment Jean Chrysostome et Ambroise de Milan (De officiis ministrorum), cités par L. Charles-DominiQue, Musiques savantes, musiques populaires..., p. 55-56.

44. Les stalles conservées à Poulaines proviennent de l'abbaye de la Vanusse qui a été détruite.

45. Toutefois la cornemuse est moins négative lorsqu'elle est aux mains des bergers, surtout lorsque ceux-ci viennent assister à la Nativité, bien qu'il y ait l'idée d'opposition entre musique céleste (des anges) et musique terrestre.

46. En flamand, la cornemuse est appelée duivelzack, littéralement «sac du diable»: $c f$. R. HAMMERSTEIn, Diabolus in Musica. Studien zur Ikonographie der Musik des Mittelalters Berne, 1974, p. 62

47. Notons que la cornemuse est aussi proscrite des mains des femmes dans l'iconographie.

48. Cf. Ovide, Métamorphoses, XI, v. 145-175. 
laquelle sont tendues des cordes attachées par des clous ${ }^{49}$. La proportion imposante d'anges jouant d'instruments à cordes par rapport à ceux jouant d'instruments à vent s'explique certainement par ce substrat symbolique relatif aux instruments de musique au Moyen Âge.

Suivant la même logique, les percussions, souvent taxées de «bruyantes ${ }^{50}$ » sont rarissimes aux mains des anges dans les stalles et les manuscrits. On trouve cependant, dans le Bréviaire à l'usage de Paris (ms. Châteauroux, BM, 2, $\mathrm{f}^{\circ} 423$ ), un ange jouant des nacaires. Or, les lettrines de ce manuscrit comportent une grande variété d'instruments, comme la chalemie (instrument «haut»), juste au-dessus de l'ange percussionniste, et la cornemuse, précédemment citée sur un autre folio; on peut en déduire alors que l'idée était certainement de présenter un instrumentarium le plus large possible, la musique des sphères utilisant tous les instruments. Il s'agit aussi peut-être d'un rapprochement avec l'ordonnancement de la musique décrit par Isidore de Séville dans son De musica ${ }^{51}$, dans lequel il élabore à la suite de Cassiodore et saint Augustin une vision tripartite de la musique: l'harmonique (concernant la voix), l'instrumentale (concernant les instruments à vent) et la rythmique (concernant les instruments à cordes et à percussion $)^{52}$. Force est de constater que lorsque les anges sont figurés en «ensembles», les instruments qu'ils pratiquent sont souvent tripartites: à vent, à cordes et à percussion (stalles d'Amiens, Somme, Picardie). Outre une cohérence proprement musicale, la réunion de ces trois types d'instruments était certainement une image de l'harmonie et de la globalité du monde qui, comme la Musica, est tripartite.

Les anges musiciens symbolisent donc l'ordre harmonique du monde dans l'iconographie des stalles et des manuscrits. Non seulement ils tiennent des instruments plutôt destinés à accompagner le chant liturgique (harpe, luth, vièle, orgue portatif, etc.) et à la symbolique positive, mais ils les utilisent aussi de façon réaliste, sans la gesticulation propre aux jongleurs ${ }^{53}$. Sur une jouée à Amiens (Somme, Picardie) (fig. 3), ils sont juxtaposés avec deux putti, l'un à cheval sur l'autre. Or, la nudité s'ajoutant

49. Cf. L. Charles-Dominique, Musiques savantes, musiques populaires... (sur le vent, chap. 8; sur l'allégorie christique des cordophones, p. 89 sq.).

50. Les percussions sont en effet les instruments privilégiés du charivari et de la «contremusique»: $c f$. L. ChARLES-DominIQue, Musiques savantes, musiques populaires..., p. $190 \mathrm{sq}$. 51. ISIDORE DE SÉvILLE, Originum sive Etymologiarum, III, XV-XXIII.

52. Notons que dans le De institutione musica de Boèce, la musica est aussi tripartite mais répartie en musica mundana, musica humana et musica instrumentalis et c'est dans cette dernière catégorie que prennent place les instruments de musique joués par les êtres humains.

53. La gesticulation des histrions et des jongleurs contant des chansons de geste est en effet réprouvée par de nombreux théologiens médiévaux qui l'opposent aux gestes mesurés du prédicateur: cf. J.-C. Schмiтt, La Raison des gestes dans l'Occident médiéval, Paris, 1990 p. $43 s q$.

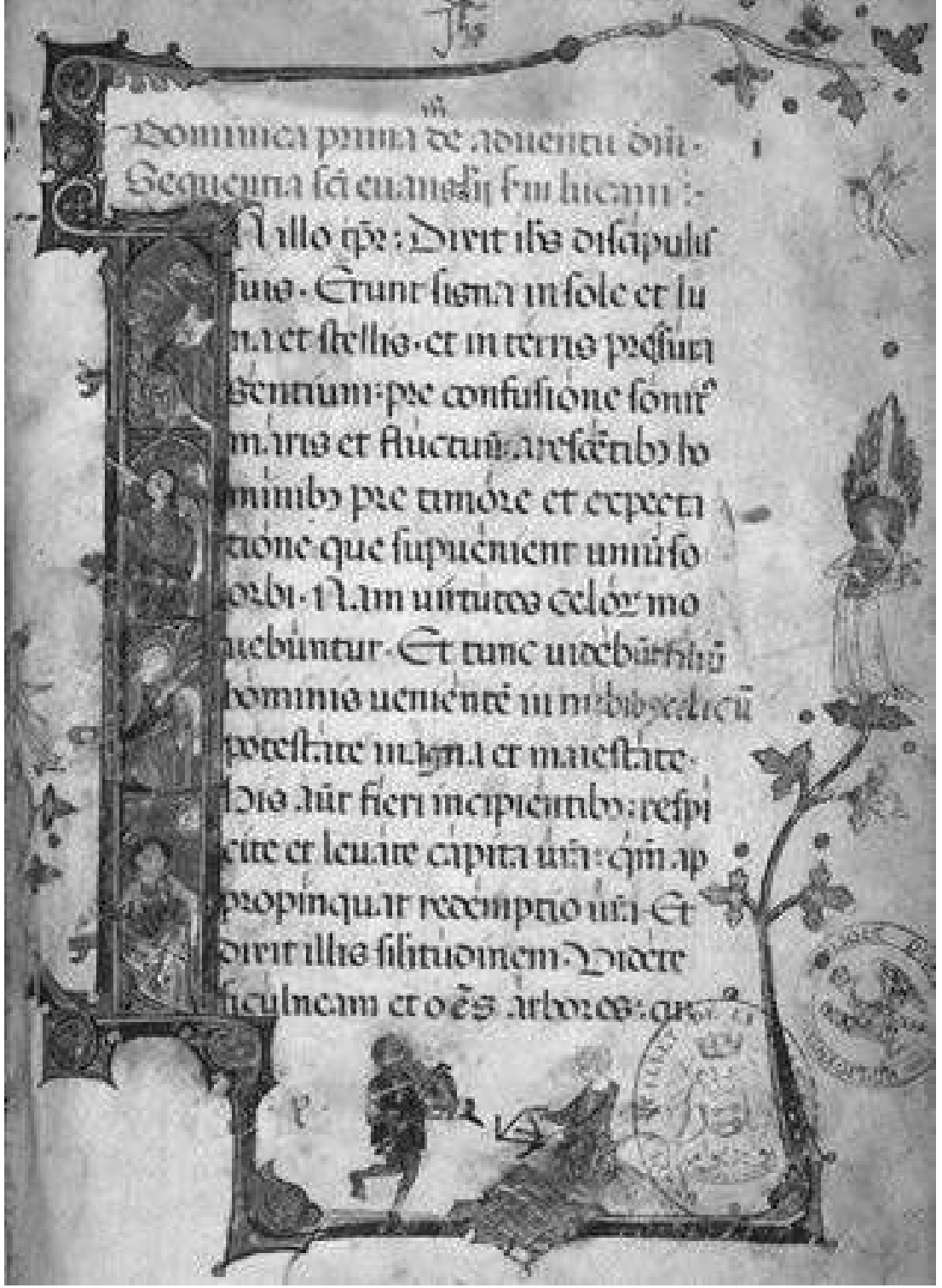

Fig. 4. Ange musicien et charivari, BM d'Avignon, ms. 24, $\mathrm{f}^{\mathrm{o}} 1$ (C) BM d'Avignon, IRHT). 
au chevauchement ${ }^{54}$ induit ici un contraste entre l'ordre représenté par les anges musiciens et le désordre de cette position mettant en valeur le corps grotesque des putti; l'harmonie est mise en regard de la disharmonie. La jouée d'Amiens est encore plus intéressante puisqu'elle oppose des chérubins nus jouant d'instruments hauts à la symbolique plutôt négative (tambour, trompe, fifre et triangle à anneaux) avec des anges portant des aubes longues jouant d'instruments bas à la symbolique positive (vièle, luth, harpe et organetto). L'opposition entre la nudité et le port d'une aube renforce encore l'idée de figuration de l'harmonie et de la disharmonie, d'autant plus que les chérubins nus joueurs de hauts instruments sont situés à gauche sur la jouée (du côté sinistre) et les anges joueurs de bas instruments à droite. Dans les marges de manuscrits, confrontés à d'autres personnages, notamment les hybrides et les animaux, ils évoquent aussi clairement l'ordre harmonieux du monde et le bon modèle à suivre. Dans l'Évangéliaire conservé à la bibliothèque municipale d'Avignon (ms. 24, fo 1) (fig. 4), un ange joue du psaltérion dans une position très digne et élégante, les ailes levées derrière la tête, alors qu'en dessous un singe utilise un soufflet comme une vièle et des pinces à feu en guise d'archet, face à un hybride entrechoquant des morceaux de métal. Le psaltérion, instrument harmonique à la symbolique christique, est opposé aux instruments détournés du charivari: les morceaux de métal entrechoqués et la «vièle singée ». L'ordre est ici clairement opposé au désordre, l'harmonie au bruit.

Si le quadrivium établit des liens étroits entre les quatre disciplines qui le constituent, les rapprochements entre musique et astronomie sont manifestes dans la pensée médiévale, parce qu'ils permettent de mieux comprendre le fait que l'harmonie musicale est aussi une musique des sphères. Cette idée semble s'exprimer à travers les images d'anges musiciens à l'époque gothique ${ }^{55}$. Outre les enluminures de manuscrits et les stalles sculptées, un exemple est fondamental, celui des fresques de la collégiale de Saint-Bonnet-le-Château (Loire, Rhône-Alpes) ${ }^{56}$ représentant des anges musiciens utilisant des instruments différents (fig. 5). Roger Cotte a été le premier à repérer une organisation zodiacale des divers

54. Aristote est ainsi représenté chevauché par Phyllis, ce qui est un avilissement total du grand philosophe, réduit à l'état d'animal (d'après le Lai d'Aristote, dont la première version est datée de 1220): $c f$. W. MulLER, «Hybrids in Choirstalls...».

55. Citons l'exemple de la première œuvre musicale inspirée de l'harmonie des sphères qui fait intervenir des anges: Naturalis concordia vocum cum planeti, datant probablement du début du XIII ${ }^{\circ}$ s., et qui comporte une gamme planétaire couvrant deux octaves, la première pour les astres, la seconde pour les séraphins, chérubins, thrônes et vertus; ms. Paris, BnF, lat. 7203, $\mathrm{f}^{\circ} 2 \mathrm{v}^{\circ}-3 ; c f$. aussi J. HANDSCHIN, «Ein mittelalterlicher Beitrag zur Lehre von der Sphärenharmonie», Zeitschrift für Musikwissenschaft, 9 (1927), p. 193-208.

56. Commandées par Louis II de Bourbon pour son épouse vers 1415-1420.

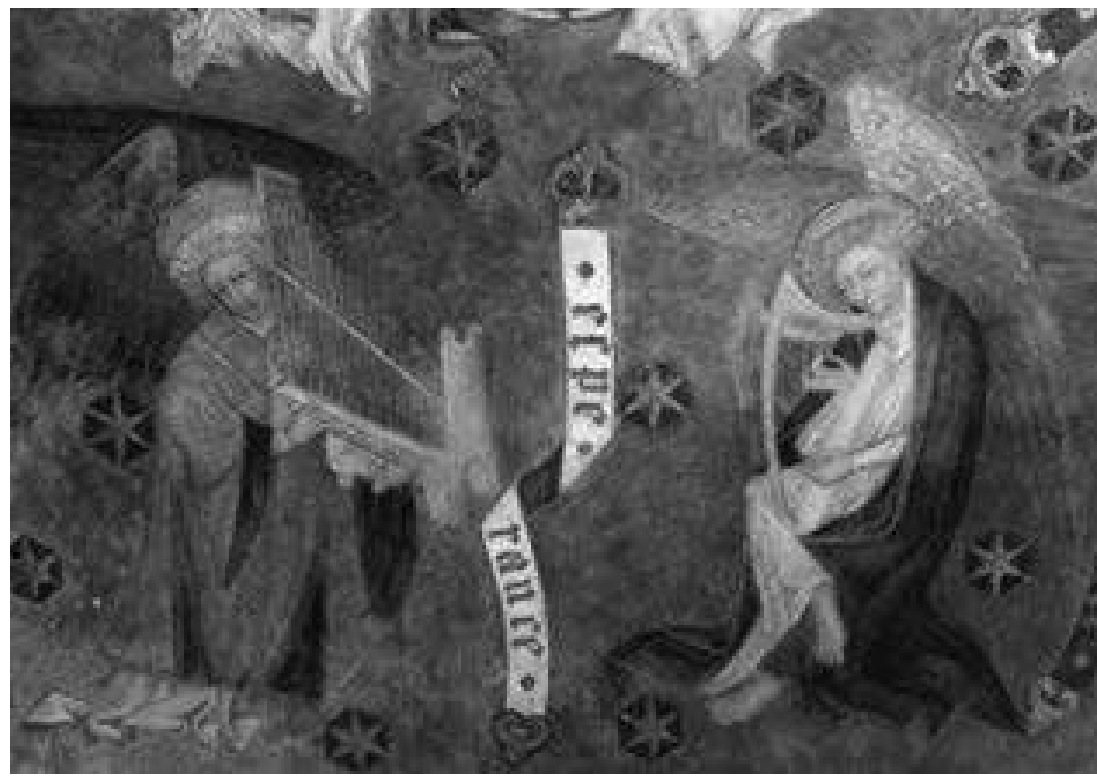

Fig. 5. Anges musiciens, fresques de la collégiale de Saint-Bonnet-le-Château (ㄷ) W. Muller)

instruments de musique représentés ${ }^{57}$ et son argumentation d'après Boèce, Martianus Capella et Pline a été reprise par Françoise Roy-Gerboud ${ }^{58}$ et Manfred Kelkel ${ }^{59}$. Sans détailler le processus d'organologie zodiacale argumenté par ces auteurs, notons que les anges musiciens de la voûte de la crypte de Saint-Bonnet-le-Château forment ensemble les constellations correspondant à une nuit de Noël des années 1420. Dans ce cas, le lien entre les anges musiciens et l'ordre harmonique du monde est évident et l'idée de modèle est encore présente puisque cette organisation des anges sur la voûte de la crypte offre un «parcours » au fidèle, dont la porte sud-ouest est le point de départ et d'arrivée ${ }^{60}$. Comme le souligne Manfred Kelkel, les fresques de Saint-Bonnet relèvent donc d'un art religieux au sens littéral du mot religare : relier la terre au ciel ${ }^{61}$

57. R. CotTe, «Une vision cosmique de Noël: la crypte de Saint-Bonnet-le-Château», Fraternité évangélique, 12 (décembre 1969), p. 77.

58. F. Roy-Gerboud, St. Bonnet-le-Château et l'harmonie des sphères, maîtrise, Université Lumière-Lyon II, 1986.

59. M. KelKel, Musique des mondes. Essai sur la métamusique, Paris, 1988, p. 105-112. 60. Ibid., schéma p. 106

61. Ibid., p. 110 
$\mathrm{Au} \mathrm{Xv} \mathrm{v}^{\mathrm{e}}$ siècle un changement intervient progressivement; les anges musiciens se font de plus en plus rares dans les enluminures de manuscrits qui seront bientôt remplacés par des imprimés. À la même époque, dans les stalles, ces anges sont l'objet d'une «récupération» par des seigneurs influents pratiquant une certaine forme de culte de la personnalité. Il ne subsiste qu'un morceau de dossier des stalles de la chapelle ducale de la Chartreuse de Champmol (conservé au musée des Beaux-Arts de Dijon, Côte d'Or, Bourgogne), dans lequel sont juxtaposés des anges musiciens et des blasons; par cet «assemblage», on peut comprendre que le duc de Bourgogne se place dans l'harmonie du monde. Celle-ci, évoquée par les anges musiciens, est ainsi utilisée pour la glorification d'une personnalité temporelle. Si par sa fonction politique le duc de Bourgogne doit effectivement suivre le modèle du roi David ${ }^{62}$ musicien et de fait être capable de faire régner l'harmonie dans le territoire qu'il gouverne, cette image s'inscrit dans un contexte d'émergence du culte de la personnalité dont Philippe le Hardi est coutumier et qu'il souhaite exprimer dans les arts dès la fin $\mathrm{du} \mathrm{XIV}^{\mathrm{e}}$ siècle. Il faut ensuite attendre le milieu du XVI ${ }^{\mathrm{e}}$ siècle pour que les anges musiciens disparaissent totalement de l'iconographie des stalles, qui comportera alors surtout des éléments décoratifs issus de l'Antiquité telles les consoles d'acanthe ou encore les fameux putti, angelots potelés et nus auxquels aucune fonction ne semble plus être attribuée. Or, cette évolution iconographique de l'ange qui perd sa capacité à agir et, de fait, ses attributs (les instruments de musique en tête) en devenant un être profondément charnel trouve un écho dans le changement des conceptions du monde entre le Moyen Âge et la Renaissance.

Les anges ont un rôle actif pour l'harmonie du monde dans les conceptions médiévales, puisqu'ils participent aux mouvements des planètes, dont la rotation produit la musique des sphères. Michel-Pierre Lerner, dans un ouvrage très complet sur les représentations cosmiques à travers les âges ${ }^{63}$, soutient qu'aucune hypothèse alternative au géocentrisme, et surtout à la doctrine des «substances spirituelles motrices des astres», n'eut de fortune auprès des médiévaux. Pourtant il y a eu des hypothèses alternatives, comme celle de Jean Buridan (1292-1363) qui émet l'idée d'une impulsion initiale: l'impetus, donné par Dieu, permettant aux orbes célestes de se mouvoir sans l'aide des anges. Cette théorie sera complètement marginalisée, au point que les propres élèves de Buridan, tel Albert de Saxe, la renièrent, tandis que les disciples de Guillaume d'Ockham réussirent à ce

62. L'objectif politique de la musique est évoqué dès Platon: $c f$. notamment N. Guidobaldi, «La musique du prince», Médiévales, 32 (1997), p. 59.

63. M.-P. LERNER, Le Monde des sphères, genèse et triomphe d'une représentation cosmique, Paris, 1996, t. I, p. 194 que ses écrits soient mis à l'Index Librorum Prohibitorum de 1474 à $1481^{64}$ Il faut attendre la fin du $\mathrm{XVI}^{\mathrm{e}}$ siècle pour que la cosmologie géocentriste éclate et voit l'émergence d'une "physique céleste» en tant que telle avec Ticho Brahé (1546-1601) et Johannes Kepler (1571-1630) dans laquelle les anges et toute autre entité spirituelle n'ont plus leur place, ébranlant ainsi les conceptions médiévales du monde, fondées sur une métaphysique céleste platonicienne ${ }^{65}$. Johannes Kepler, l'un des savants les plus représentatifs de cette période charnière, changea d'ailleurs d'avis sur l'organisation du monde au cours de sa vie. Ainsi, dans son Mysterium Cosmographicum (1595), il commença par expliquer les mouvements des planètes par la force des anges qui les meuvent (opinion «médiévale»); puis, il écrivit dans l'Epitome (vers 1630) qu'il n'est pas nécessaire de recourir à des anges, là où l'action de forces matérielles ou semi-matérielles, telles que la lumière ou le magnétisme, offre une explication suffisante; le «mécanisme» suffit alors parce que les mouvements planétaires suivent des lois mathématiques ${ }^{66}$.

Les anges musiciens sculptés dans les stalles et peints dans les manuscrits sont donc les expressions personnifiées de l'ordre harmonique $\mathrm{du}$ monde, universel, divin, cosmologique propre au Moyen Âge; ils tiennent plutôt des instruments de musique à cordes, non seulement parce que ceux-ci permettent d'accompagner le plain-chant et qu'ils sont porteurs d'une symbolique christique, mais aussi parce que ces instruments ont une place privilégiée dans les conceptions médiévales du monde. Reprenant le point de vue de certains auteurs antiques, Boèce opère des analogies entre les cordes et les corps célestes (De institutione musica, I, xxvii). Et les anges musiciens disparaissent de la sculpture des stalles en même temps que sont émises et admises des hypothèses les excluant du bon fonctionnement du monde. Dès le milieu du XVI ${ }^{\mathrm{e}}$ siècle, ils sont en effet remplacés par ces putti potelés n'ayant plus de fonction (ne tenant plus ni phylactère, ni instrument de musique), parfois réduits à leur plus simple expression: une tête encadrée d'ailes.

L'harmonie du monde est donc bien représentée dans l'art médiéval en général et dans les stalles et les enluminures de manuscrits gothiques en France en particulier. La période charnière avec la Renaissance montre un

64. Sur Jean Buridan, cf. G. Kuma, John Buridan, Oxford/New York, 2009, J. M. M. H. ThiJssen \& J. ZuPKo, The Metaphysics and Natural Philosophy of John Buridan, Leyde, 2001.

65. Notons que les théories que Nicole Oresme et Nicolas de Cues ont forgées aux $\mathrm{XIV}^{\mathrm{e}}$ et $\mathrm{XV}^{\mathrm{e}} \mathrm{s}$. présentent une vision très complexe du mouvement des orbes qui ne peut être réduite à une «simple» métaphysique céleste platonicienne : $c f$. M.-P. LerNER, Le Monde des sphères..., II, p. 86-88 et p. 91-94

66. Cf. A. KoYré, «Apport scientifique de la Renaissance», dans Etudes d'histoire de la pensée scientifique, Paris, 1973, p. 56; ID., Chute des corps et mouvement de la terre de Kepler à Newton, Paris, 1973, chap. 2. 
basculement entre les conceptions du monde géocentrique et héliocentrique, et les anges musiciens disparaissent de l'iconographie en même temps que le ciel se vide de ses créatures spirituelles qui permettaient aux orbes de se mouvoir. La figure du roi David accordant sa harpe n'est pas seulement l'évocation d'une conception du monde, mais porte aussi en elle le modèle à suivre pour retrouver cette harmonie en devenant un nouvel être humain, en parfaite harmonie avec le monde ${ }^{67}$. Et c'est par la pratique du chant liturgique que cet ordre harmonique du monde est mis à la portée des fidèles. En ce qui concerne les représentations d'anges musiciens, outre leur évolution iconographique sensible en relation avec les changements de conceptions du monde, leur juxtaposition fréquente à des hybrides ou des animaux musiciens est remarquable. L'harmonie est alors mise en regard de la disharmonie, l'ordre face au désordre; le modèle de l'harmonie étant renforcé par sa comparaison avec la disharmonie. La vision d'un monde ordonné est alors mise en exergue, grâce au parallèle entre les anges musiciens, véritables acteurs de la musique du monde, supplantant les animaux ou les hybrides produisant plutôt du bruit qu'une musique.

Welleda MULLER - Université Paris-Sorbonne, ANR Musiconis (http://musiconis.paris-sorbonne.fr)

\section{Figures de l'harmonia mundi dans les manuscrits et les stalles gothiques} n France.

L'harmonia mundi est une donnée essentielle de la culture normée du Moyen Âge. L'idée que le monde est régi par un ordre divin et que la rotation des sphères produit un son harmonique inaudible aux oreilles humaines est présente à tous les niveaux de la société et chez la majorité des intellectuels; mais cette idée n'est pas uniquement débattue dans des textes par les théoriciens, elle est aussi représentée en images. Ainsi, la multitude d'anges musiciens jouant majoritairement $d$ 'instruments a cordes est certainement l'image de la musique des spheres, en particulier dans les stalles et les manuscrits, deux supports artistiques étroitement liés avec le chant liturgique dont la pratique permet de retrouver l'harmonie divine de la Création. Á côté des anges musiciens, images de l'harmonia mundi, un modèle est présenté pour encourager les fidèles dans leur propre recherche de l'harmonie: il s'agit du roi David accordant sa harpe, mettant ainsi en lumière la nécessité de réglage de l'ordre du monde. Toutefois, les $X V^{e}$ et $X V l^{e}$ siècles sont une période charnière durant laquelle vont évoluer les conceptions du monde, ce qui transparaît dans les images. Les anges musiciens disparaissent en effet progressivement de la sculpture Les anges musiciens disparaissent en effet rrogressiemer des putti de chair n'ayant plus de fonction, en même temps que les cieux se vident des que les cieux se

musique des sphères - roi David - anges musiciens - stalles de chœur manuscrits enluminés

67. Dépouillé du vieil homme évoqué par saint Paul et étudié à la Renaissance par C. SCHWENCKFELD: Homme charnel, homme spirituel, trad. A. SCIEGIENNY, Wiesbaden, 1975.
Representations of the harmonia mundi in Gothic Manuscripts and Choir Stalls in France. King David Tuning his Harp and Angel Musicians

Harmonia mundi is an essential notion in traditional medieval culture. The dual idea that the world is governed by a divine order and that the rotation of the spheres produces a harmonic sound inaudible to human ears is present throughout medieval society and for the intellectuals in particular. This idea is not only discussed in texts by scholars, but it is also represented in pictures. Thus, the multitude of angelic musicians mainly playing stringed instruments is certainly the image of the music of the spheres, especially in the choir stalls and manuscripts, two artistic media linked to the liturgical chant whose practice allows find the divine harmony of Creation. Besides the angel musicians, images of harmonia mundi, a model is presented to encourage the faithful in their own quest for harmony: it is King David tuning his harp, thus highlighting the need for adjustment the word order. However, the fifteenth and sixteenth centuries were a turning point that saw a chang in worldviews, which is reflected in the imagery. The angelic musician disappeared gradually from the carved choir stalls and painted manuscripts to be replaced by putti who have no function; at the same time, the heavens were empty of spiritual substances which allowed orbs to move in medieval conceptions of the world.

music of the spheres - King David - angel musicians - choir stalls illuminated manuscripts 